\title{
Determination of methanol residues in crude glycerol for animal feed by gas
}

\section{chromatography}

\author{
Carina Nazato®, Alessandra de Cássia Romero*(), Adibe Luiz Abdalla®
}

Universidade de São Paulo/CENA - Lab. de Nutrição Animal, Av. Centenário, 303 - 13416-000 - Piracicaba, SP - Brasil. *Corresponding author <acromero@usp.br>

Edited by: Gerson Barreto Mourão

Received October 27, 2017

Accepted June 25, 2018

\begin{abstract}
Crude glycerol is a major by-product of biodiesel production and is an economical additive feed for ruminants. However, residual methanol in crude glycerol can be harmful to animal health. Several methods exist for quantifying methanol residues in biodiesel, yet few have been described that identify the methanol level in crude glycerol. We propose a method for determining the methanol level in crude glycerol destined for animal feed. Crude glycerol was extracted from the headspace and quantified by gas chromatography using a flame ionization detector (GC-FID). The method was linear up to $0.5 \%$ of methanol. The limits of detection (LOD) and quantification (LOQ) were 0.015 and $0.031 \%$, respectively. No significant matrix effects were observed. Precision was $2 \%$ at the 0.05 and $0.5 \%$ levels. The average percentage of recovery was $90 \%$. Three analyzed samples of crude glycerol had methanol residues of 0.027-7.802\%. Furthermore, this methanol quantification method may be externally or internally calibrated using a GC manual injector. A reduction of at least $20 \%$ in running time was obtained with good resolution between the peaks. Thus, this method can be applied to determine the methanol level in crude glycerol according to the upper limits for animal feed (5,000 ppm) and for human consumption $(150 \mathrm{ppm})$. Finally, this method is useful for the quality control of crude glycerol intended for use in animal feed, enabling the alternative use of this by-product to add value to the biodiesel production chain.
\end{abstract}

Keywords: co-product, feedstuff, biodiesel, toxic compound, ruminant

\section{Introduction}

Crude glycerol is a major by-product of biodiesel production and it is usually further refined to obtain a pure material that is used by the pharmaceutical and cosmetic industries. However, glycerol purification is a laborious and expensive process, and only largescale industrial refinement processes are cost-effective (Xiao et al., 2012). As an alternative to reduce cost, crude glycerol has been increasingly destined for use in industrial feedstock instead of undergoing further refinement processes. A number of authors state that the inclusion of glycerol from biodiesel production in animal feed is one way of reducing methane emission. Furthermore, glycerol in animal diets increases the efficiency of energy usage by animals without any adverse effects (Lee et al., 2011; Wilbert et al., 2013). However, these claims are not fully supported by the literature (Boyd et al., 2013; Ezequiel et al., 2015), implying that the remaining levels of chemical residues in crude glycerol generated from the biodisel industry should be further tested (Paiva, 2015).

Crude glycerol composition depends on the industrial process used to produce biodiesel; however, methanol and soap are major contaminants commonly found in this by-product (Sivasankaran et al., 2016). Methanol is a toxic compound, even for ruminant animals, and its residues in crude glycerol destined for animal feed have not been sufficiently evaluated.

Ruminants are less susceptible to toxicity than monogastric animals because the ruminal microbiota is capable of detoxifying toxic compounds. However, the resistance of ruminants is not uniform across all species and depends on the individual ruminal microbiota and feed composition (Smith, 1992).

The Association of American Feed Control (AAFCO) approved in 2015 an allowable limit of up to 5,000 ppm of methanol in crude glycerol from biodiesel production (Wilkinson, 2017). This limit is more realistic than the previous FDA methanol limit established for human nutrition (150 ppm), which had also been used for animal feed until 2006 (FDA, 2014).

The European Committee for Standardization describes a gas chromatography method (BS EN 14110:2003) (CEN, 2003) to measure methanol residues in fatty acid methyl esters (FAME) for use as diesel fuel. However, to our knowledge, few similar gas chromatography (GC) methods for quantifying methanol in crude glycerol have been validated or published (Shuai et al., 2013). Herein we propose a simple methodology for quantifying methanol residues in crude glycerol by gas chromatography.

\section{Materials and Methods}

\section{Materials and chemicals}

The reagents methanol and 2-propanol were HPLC-grade, and glycerol (G9012, Sigma-Aldrich ${ }^{\circledR}$ ) $\geq 99 \%$ pure. A GC Shimadzu gas chromatograph (model GC 2014) equipped with a flame ionization detector (FID) and a Stabilwax ${ }^{\circledR}$-DA column (15 m $\times 0.53 \mathrm{~mm}$, ID $\times 1 \mu \mathrm{m}$, Restek, part number 11052) was used and con- 
nected to the GC Solution software program (Shimadzu). A sample lock syringe for gases was used to perform manual injections. A THELCO model 85 water bath, a Retilínea drying oven, and a QH Kerry ultrasound bath were used for preparing the samples.

\section{Crude glycerol samples}

Three samples of crude glycerol from biodiesel production were analyzed, two samples were obtained from the biodiesel industry, and another by the transesterification of Jatropha curcas oil (Maciel et al., 2013).

\section{Preparation of calibration solutions and samples}

The suitability of the BS EN 14110:2003 method to quantify methanol in biodiesel was tested for crude glycerol. The BS EN 14110:2003 method relies on the use of automatic headspace equipment wherein methanol is quantified by external calibration; or, if a manual system is used, an internal calibration procedure can be performed. We verified the suitability of the BS EN 14110:2003 manual injection procedure for crude glycerol. Firstly, three calibration solutions were prepared and injected. The acceptance criterion was that the average of the injected solutions (in triplicate) must have a coefficient of variability (CV) no greater than $15 \%$. The original method failed to pass this acceptance criterion based on the $\mathrm{CV}$ of the calibration solutions prepared from glycerol. Thus, this methodology was modified and evaluated for crude glycerol considering the BS EN 14110:2003 criteria (CEN, 2003), and subsequent validation tests were performed.

Three calibration solutions of methanol in glycerol were prepared at 0.5 (solution A), 0.1 (B), and $0.01 \%$ (C). For solution A, $200 \mu \mathrm{L}$ of methanol was added to a volumetric flask containing $25 \mathrm{~mL}$ of glycerol. Solution B was prepared by diluting $6.25 \mathrm{~g}$ of solution A in $25 \mathrm{~mL}$ of glycerol. Solution $\mathrm{C}$ was obtained by diluting $1.25 \mathrm{~g}$ of solution B in $10 \mathrm{~mL}$ of glycerol. The volumetric flasks were sealed with parafilm immediately after preparation until extraction. The volumetric flasks were manually agitated and sonicated in an ultrasound bath with ice for $3 \mathrm{~min}$ to prevent heterogeneity in the distribution of methanol in the glycerol solutions, which could result in measurement errors, as previously observed. Prior to extraction and injection, $5 \mathrm{~g}$ of each calibration solution was transferred $(\mathrm{w} / \mathrm{w})$ to vials of $20 \mathrm{~mL}$. Five $\mu \mathrm{L}$ of 2-propanol was added, and all solutions were sealed with parafilm and sonicated in an ultrasound bath with ice for $3 \mathrm{~min}$. The methanol extraction was performed using the static headspace technique at $80^{\circ} \mathrm{C}$ for $45 \mathrm{~min}$, and $100 \mu \mathrm{L}$ of its gas was collected in the headspace for subsequent injections.

The calibration factor was obtained by calculating the ratio of the concentration of methanol multiplied by the peak area of 2-propanol (internal standard, added before extraction) to the concentration of 2-propanol multiplied by the peak area of methanol (CEN, 2003).

\section{Extraction of methanol in crude glycerol}

Five grams of crude glycerol was weighed in a septum vial $(20 \mathrm{~mL})$ to which $5 \mu \mathrm{L}$ of 2 -propanol was added before methanol was extracted according to the static headspace technique described as follows. The sample was homogenized, and the vial with crude glycerol was held at $80{ }^{\circ} \mathrm{C}$ for $45 \mathrm{~min}$ in a water bath. Then, 100 $\mu \mathrm{L}$ of gas in the headspace was collected with a gas-tight syringe pre-heated in the oven at $60{ }^{\circ} \mathrm{C}$. This aliquot was immediately injected into the GC by manual injection.

\section{Chromatographic analysis}

The methanol elution was evaluated by temperature-programmed gas chromatography. The temperature was set at $40{ }^{\circ} \mathrm{C}$ for $1.2 \mathrm{~min}$ followed by a heating level of $20{ }^{\circ} \mathrm{C} \mathrm{min}{ }^{-1}$ for up to $1.7 \mathrm{~min}$, until the temperature reached $50{ }^{\circ} \mathrm{C}$ where it remained until the end of the running sequence. The carrier gas was

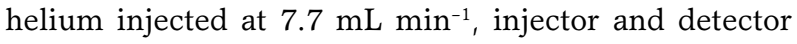
temperatures were 100 and $250{ }^{\circ} \mathrm{C}$, respectively. The sample was injected in the splitless mode. A volume of $100 \mu \mathrm{L}$ was injected, and total running time was $4 \mathrm{~min}$.

\section{Validation of method}

In addition to the internal standard calibration procedure, the method was validated according to the following parameters: linearity, matrix effects, limits of detection (LOD) and quantification (LOQ), recovery, and precision (repeatability) (Magnusson and Örnemark, 2014). Linearity was evaluated by regression of the analytical curve. Seven solutions were prepared by adding methanol to pure glycerol (blank samples free of methanol and other contaminants); each solution was injected seven times into the gas chromatograph. The tested levels of methanol were $0,0.01,0.05,0.10,0.20$, 0.30 , and $0.50 \%$. The matrix effects were investigated, comparing the slope of the analytical curve with that of the matrix-matched curve (Economou et al., 2009). The matrix-matched curve was prepared by adding the same seven levels of methanol of the analytical curve to samples of crude glycerol without detectable residues of methanol. Each solution was injected seven times into the gas chromatograph.

The limit of detection (LOD) was obtained based on the signal-to-noise ratio approach ( $\mathrm{ICH}, 2005)$. The extraction efficiency of the methodology was evaluated by recovery tests at levels of $0.50,0.20$, and 0.05 $\%$ methanol. The precision was estimated by calculating the coefficients of variation between injections for two levels of methanol (0.05 and $0.50 \%)$. Solutions were prepared for the analytical and matrix-matched curves, and recovery and precision tests were performed by sealing the flasks with parafilm immediately after adding the prescribed levels of methanol in glycerol. All solutions were manually agitated and sonicated in an ultrasound bath with ice for $3 \mathrm{~min}$, as described in the section on the preparation of the calibration solutions. 


\section{Application of the developed method in industrial samples}

Three samples of crude glycerol from biodiesel production were analyzed to verify whether the chromatographic profile had changed as a result of the behavior of the co-eluted compounds. Five grams of crude glycerol was weighed in vials, to which $5 \mu \mathrm{L}$ of the internal standard was added. Extractions were then performed as previously described.

\section{Results and Discussion}

\section{Preparation of calibration solutions and extraction of samples}

The BS EN 14110:2003 method used for the methanol analysis of biodiesel samples was tested for its applicability to measuring the methanol content of glycerol. For this method to be suitable, a CV $\leq 15 \%$ between prepared calibration solutions must be shown; however, the tests for glycerol showed a CV > $30 \%$ based on the average contents of the solutions. Therefore, the BS EN 14110:2003 method is not suitable for the methanol analysis of crude glycerol.

The obtained CV values for crude glycerol were probably due to the heterogeneity of methanol in the sequential glycerol dilutions. In addition, the difference in viscosity between biodiesel and glycerol likely enhances the heterogeneity of the distribution of methanol artificially added to glycerol.

To improve the homogeneity of the solutions, manual agitation followed by sonication was carried out, as described in BS EN 14110:2003. Calibration solutions were sealed with parafilm immediately after the addition of methanol. This procedure was adopted to avoid the loss of volatile compounds by evaporation both in the calibration solution and in the samples. Specifically, volumetric flasks were manually agitated and sonicated in an ultrasound bath with ice for $3 \mathrm{~min}$. The method for determining methanol in biodiesel does not require these procedures, probably because of the physical properties of biodiesel, such as viscosity. However, biodiesel viscosity may vary as a function of temperature, oil source fatty acid profile, carbon chain length, and degree of saturation; also, comparatively, glycerol is more viscous than biodiesel (Patzer, 2007; Refaat, 2009). This difference in viscosity might explain why methanol dispersion is better in biodiesel than in glycerol. Thus, it is possible the sonication step improves the dispersion of methanol in glycerol by shearing, reducing the viscosity and promoting methanol dispersion. Improvements in the uniformity of the distribution of chemical species in ultrasound baths have already been described in the literature (Ohsaka et al., 2016). However, despite the sonication process improving the methanol dispersion, it may warm the metal cover and glass vial material, volatilizing the methanol. Thus, the cold-water ultrasonic bath and sealing of the flask were performed to avoid leakage of methanol and 2-propanol (internal standard in samples).
The CV observed between calibration solutions was $6 \%$, confirming that the modified method is suitable for quantifying methanol content in crude glycerol. Specifically, this procedure is suitable for quantifying methanol in crude glycerol according to the internal standard method, as described in the BS EN 14110:2003 method for quantifying methanol in biodiesel. Thus, the BS EN 14110:2003 procedures were validated and adopted herein for the analysis of crude glycerol samples.

\section{Chromatographic analysis}

Methanol had a retention time (Rt) of $2.3 \mathrm{~min}$, and the internal standard (2-propanol) has a retention time of 2.6 min during the developed chromatographic separation (Figure 1).

The resolution between methanol and 2-propanol was 1.8. This resolution was suitable as the minimum value for complete separation between two peaks is 1.5 (McNair and Miller, 2009). No interfering peaks were observed in the chromatograms of crude glycerol, nor was any evidence of contamination by parafilm observed. The total running time of $4 \mathrm{~min}$ is faster than the BS EN 14110:2003 method for quantifying methanol in biodiesel.

\section{Validation of method}

The method developed was evaluated based on linearity, matrix effects, limits of detection (LOD) and quantification (LOQ), recovery, and precision. A linear response was observed given the correlation coefficient $\left(\mathrm{R}^{2}=0.99\right)$ of the analytical and matrix-matched curves. The matrix effects test predicts the influence of other compounds present in the sample. The occurrence of matrix effects can cause errors in the measurement of the analyte due to suppression or enhancement of peak signals (ISO, 2002). The occurrence of matrix effects was checked by calculating the ratio of the angular coefficient of the curves in the solvent (pure glycerol) to those in the matrix (crude glycerol). No significant matrix effects were observed. The value of the observed matrix effects $(\mathrm{C} \%)$ was $4 \%$, which is within the range considered to be characteristic of a low matrix effect $(-20 \%<$ C \% $<20 \%$ ) (Economou et al., 2009).

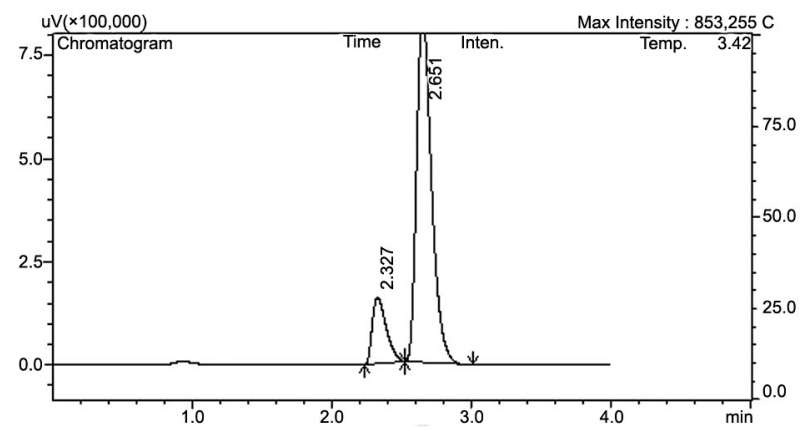

Figure 1 - Chromatogram of methanol and 2-propanol peaks in pure glycerol (standard) (Retention times of 2.3 and $2.6 \mathrm{~min}$, respectively). 
The LOD is the minimum amount of analyte that can be reliably detected in an analysis, and the LOQ is the lowest concentration of analyte that can be reliably obtained in the analysis $(\mathrm{ICH}, 2005)$. The LOD of methanol was $0.01 \%$, and the LOQ $0.03 \%$. The present methodology is therefore suitable for the detection of methanol at the levels recommended by the FDA for human consumption (FDA, 2014) and for quality control of crude glycerol intended for animal nutrition. The recovery test provided information about the efficiency of extraction. The average recovery percentage was 91 $\%$ for $0.50 \%$ added methanol, $95 \%$ for $0.2 \%$ added methanol, and $88 \%$ for $0.05 \%$ added methanol. These values are within the acceptable recovery ranges for the corresponding concentrations. Precision (RSD), as measured by the repeatability of the methodology, was high at both tested levels and the value obtained for $0.05 \%$ and $0.50 \%$ of methanol was 2 (González et al., 2010).

\section{Application of the developed method in industrial samples}

The peak profiles of the chromatograms of the industrial samples showed no changes in peak number or retention time. No interfering or co-eluted compounds were observed (Figure 2) near methanol retention time, showing that the chromatography system developed is useful for measuring methanol in crude glycerol.

To co-validate the results for methanol residues in industrial samples of crude glycerin, the methanol concentration obtained by external calibration was compared with that obtained by internal calibration (CEN, 2003), as shown in Table 1. The agreement between values was quantified by analytical curves and the calibration factor, confirming the suitability of the methodology developed in terms of accuracy.

Few studies have measured the methanol content of crude glycerol, although several previous studies have described levels of $<0.01 \%$ up to $1.15 \%$ in crude glycerol samples in the United States (Dasari, 2007) and 0.33$8.7 \%$ in Brazil (Françozo et al., 2013; Lage et al., 2014). In comparison with the values reported in the literature, the industrial samples analyzed herein had methanol levels higher than the limit recommended for human consumption (0.015\%) established by the FDA (FDA, 2014). In addition, the laboratory sample had a methanol content higher than the FDA and AFIA limits for inclusion in animal feed $(5,000 \mathrm{ppm}$, corresponding to $0.50 \%)$. The sample of crude glycerol produced from Jatropha curcas oil probably had a high methanol content because the employed process was similar to that used for biodiesel production.

Notably, only one case of toxicity in ruminants induced by methanol has been reported in the literature. In 1982, suspected cattle poisoning by methanol was reported. The level of methanol orally ingested by animals was probably much higher than the limit recommended by the FDA since analysis of the rumen fluid of the animals involved in this incident showed a

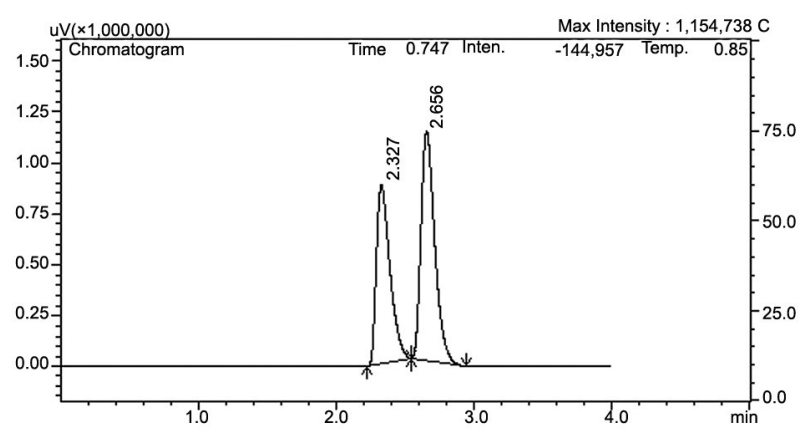

Figure 2 - Chromatogram of methanol and 2-propanol peaks in crude glycerol industrial sample (Retention times of 2.3 and 2.6 min, respectively).

Table 1 - External calibration (analytical curve) vs. internal calibration (BS EN 14110:2003) methanol concentration in crude glycerol samples from biodiesel production.

\begin{tabular}{lcc}
\hline \multirow{2}{*}{ Samples } & \multicolumn{2}{c}{ Methanol residues (\%) } \\
\cline { 2 - 3 } & Internal standard & External standard \\
\hline Industrial biodiesel 1 & 0.025 & $0.027^{1}$ \\
Industrial biodiesel 2 & 0.045 & 0.047 \\
Laboratory biodiesel 3 & 7.914 & 7.802 \\
\hline 1Numerical &
\end{tabular}

${ }^{1}$ Numerical value by external calibration in order to compare the concentration with internal standards, although, according to established acceptable limits $(\mathrm{LOD}=0.01 \%$ and $\mathrm{LOQ}=0.03 \%$ ), the values of industrial biodiesel 1 must be reported as "traces".

remaining methanol content of approximately $370 \mathrm{mg} \%$ (Rousseaux et al., 1982).

In this study, we did not intend to characterize the residual methanol in industrial samples of crude glycerol because the number of samples was insufficient for this purpose. However, we hope that the method described herein may encourage further studies to describe the methanol residue profile of industrial samples intended for animal feed.

\section{Conclusions}

The present method is suitable for determining methanol levels in crude glycerol according to the established FDA limit for human nutrition and the AAFCO recommendation for animal feed. External or internal calibration using a GC manual injector is suitable for this method of methanol quantification. This method is useful for the quality control of crude glycerol intended for animal feed and enables the alternative use of this byproduct to add value to the biodiesel production chain.

\section{Acknowledgments}

We would like to thank the Fundação de Amparo à Pesquisa do Estado de São Paulo (FAPESP; 13/10359-6; 13/10630-1) and the Coordenação de Aperfeiçoamento de Pessoal de Nivel Superior/Programa de Excelência Acadêmica (CAPES/PROEX; 33002061001P3) for financially supporting this study. 


\section{Authors' Contributions}

Conceptualization: Abdalla, A.L. Data acquisition: Nazato, C.; Romero, A.C. Data analysis: Nazato, C.; Romero, A.C. Design of methodology: Abdalla, A.L.; Romero, A.C. Writing and editing: Nazato, C.; Romero, A.C.; Abdalla, A.L.

\section{References}

Boyd, J.; Bernard, J.K.; West, J.W. 2013. Effects of feeding different amounts of supplemental glycerol on ruminal environment and digestibility of lactating dairy cows. Journal of Dairy Science 96: 470-476.

Dasari, M. 2007. Crude glycerol potential described. Feedstuffs 79: 1-3.

Economou, A.; Botitsi, H.; Antoniou, S.; Tsipi, D. 2009. Determination of multi-class pesticides in wines by solidphase extraction and liquid chromatography-tandem mass spectrometry. Journal of Chromatography A1216: 5856-5867.

European Committee for Standardization [CEN]. 2003. BS EN 14110:2003: Fat and Oil Derivatives. Fatty Acid Methyl Esters (FAME). Determination of Methanol Content. BSI Publications, Brussels, Belgium.

Ezequiel, J.M.B.; Sancanari, J.B.D.; Machado Neto, O.R.; Silva, Z.F.; Almeida, M.T.C.; Silva, D.A.V. 2015. Effects of high concentrations of dietary crude glycerol on dairy cow productivity and milk quality. Animal Feed Science and Technology 98: 8009-8017.

Food and Drug Administration [FDA]. 2014. Code of Federal Regulations, Title 21, 6, CFR573.640. Available at: http:// www.accessdata.fda.gov/scripts/cdrh/cfdocs/cfcfr/CFRSearch. $\mathrm{cfm} ? \mathrm{fr}=573.640$ [Accessed July 14, 2017]

Françozo, M.C.; Prado, I.N.; Cecato, U. 2013. Growth performance, carcass characteristics and meat quality of finishing bulls fed crude glycerol-supplemented diets. Brazilian Archives of Biology and Technology 56: 327-336.

González, A.G.; Herrador, M.A.; Asuero, A.G. 2010. Intralaboratory assessment of method accuracy (trueness and precision) by using validation standards. Talanta 82: 1995-1998.

International Conference on Harmonisation [ICH]. 2005. ICH Harmonised Tripartite Guideline Validation of Analytical Procedures: Text and Methodology Q2(R1). ICH, Geneva, Switzerland.

International Organization for Standardization [ISO]. 2002. In vitro Diagnostic Systems. Measurement of Quantities in Samples of Biological Origin. Description of Reference Materials. ISO 15194:2002. ISO, Geneva, Switzerland.

Lage, J.F.; Paulino, P.V.R.; Pereira, L.G.R.; Duarte, M.S.; Valadares Filho, S.C.; Oliveira, A.S.; Souza, N.K.P.; Lima, J.C.M. 2014. Carcass characteristics of feedlot lambs fed crude glycerol contaminated with high concentrations of crude fat. Meat Science 96: 108-113.

Lee, S-Y.; Lee, S-M.; Cho, Y-B.; Kamb, D-K.; Lee, S-C.; Kim, C-H.; Seo, S. 2011. Glycerol as a feed supplement for ruminants: in vitro fermentation characteristics and methane production. Animal Feed Science and Technology 166-167: 269-274.
Maciel, P.B.; Barros, L.L.S.; Duarte, E.C.M.; Harder, M.N.C.; Bortoleto, G.G.; Abreu Junior, C.H.; Villanueva, F.C.A. 2013. Determination of nutrients and potentially toxic elements in Jatropha curcas seeds, oil and biodiesel using inductively coupled plasma mass spectrometry. Journal of Nuclear Chemistry 297: 209-213.

Magnusson, B.; Örnemark, U. 2014. Eurachem guide: the fitness for purpose of analytical methods. A laboratory guide to method validation and related topics. Available at: http://www. eurachem.org [Accessed Feb 20, 2018]

McNair, H.M.; Miller, J.M. 2009. Basic Gas Chromatography. John Wiley, Hoboken, NJ, USA.

Ohsaka, T.; Goto, Y.; Sakamoto, K.; Isaka, M.; Imabayashi, S.; Hirano, K. 2010. Effect of intensities of ultrasound sonication on reduction of crack formation and surface roughness in iridium electrodeposits. Transactions of the Institute of Metal Finishing 88: 204-208.

Paiva, P.G.; Del Valle, T.A.; Jesus, E.F.; Betteroa, V.P.; Almeida, G.F.; Bueno, I.C.S.; Bradfordd, B.J.; Rennó, F.P. 2015. Effects of crude glycerol on milk composition, nutrient digestibility and ruminal fermentation of dairy cows fed corn silage-based diets. Animal Feed Science and Technology 212: 136-142.

Patzer, R. 2007. Stack emissions evaluation: combustion of crude glycerol and yellow grease in an industrial fire tube boiler. Agricultural Utilization Research Institute, Marshall, MN, USA. Available at: http://www.auri.org/wp-content/assets/ legacy/research/Glycerin\%20Report\%20Final.pdf [Accessed Sept 22, 2017]

Refaat, A.A. 2009. Correlation between the chemical structure of biodiesel and its physical properties. International Journal of Environmental Science Technology 6: 677-694.

Rousseaux, C.R.; Audette, R.J.; Ellefson, G. 1982. Methyl alcohol toxicity in cattle. The Canadian Veterinary Journal 23: 252.

Shuai, G.; Chunli, G.; Cuiyan, X.; Yong, F.; Yue, Z.; Xin, X.; Yixin, J. 2013. Determination of methanol content in the crude glycerol. Chemical Industrial Times 2013: 7.

Sivasankaran, C.; Ramanujamb, P.K.; Balasubramanianc, B.; Manid, J. 2016. Recent progress on transforming crude glycerol into high value chemicals: a critical review. Biofuels. DOI: 10.1080/17597269.2016.1174018

Smith, G.S. 1992. Toxification and detoxification of plant compounds by ruminants: an overview. Journal of Range Management 45: 25-30.

Wilbert, C.A.; Prates, E.R.; Barcellos, J.O.J.; Schafhäuser, J. 2013. Crude glycerol as an alternative energy feedstuff for dairy cows. Animal Feed Science and Technology 183: 116-123.

Wilkinson, L. 2017. Biodiesel derived glycerin. American Feed Industry Association (AFIA). Available at: http://www.afia.org/ article_content.asp?article $=37$ [Accessed Aug 10, 2017]

Xiao, Y.; Xiao, G.; Varma, A. 2012. A universal procedure for crude glycerol purification from different feedstocks in biodiesel production: experimental and simulation study. Industrial \& Engineering Chemistry Research 52: 14291-14296. 